\title{
Full-order observers for linear fractional multi-order difference systems
}

\author{
M. WYRWAS* \\ Faculty of Computer Science, Bialystok University of Technology, Wiejska 45A St., 15-351 Białystok, Poland
}

\begin{abstract}
The paper is devoted to the construction of observers for linear fractional multi-order difference systems with Riemann-Liouvilleand Grünwald-Letnikov-type operators. Basing on the Z-transform method the sufficient condition for the existence of the presented observers is established. The behaviour of the constructed observer is demonstrated in numerical examples.
\end{abstract}

Key words: difference operators, fractional order difference system, observer.

\section{Introduction}

Recently, there is a large increase in interest of fractional calculus application as well theoretical and practical points of view, see for example [1-12]. Basic information, ideas and some applications of fractional calculus can be found for example in $[5,13,14]$. In the paper we focus on systems with forward $h$-difference operators, so we restrict our consideration to the discrete fractional calculus that was initiated in [15]. This work found its continuation in [8, 16-19]. Recently, nabla and delta derivatives are defined and generalized to fractional orders, obtaining two formulations that are discrete versions of the Grünwald-Letnikov derivatives, see [20]. Modern technical applications and signals force to discretization of the considered systems and as the consequence a replacement of derivatives by differences with small enough steps. The main reason of considering forward difference operators is that the delta-domain approach has been promoted as an effective tool for dynamic system modeling and control. In [21] the authors demonstrate that the numerical properties of structure detection are improved by a delta-domain model and such delta-domain models provide models closely linked to the continuous-time systems. Additionally, the delta-domain approach allows to address the question of preservation of the system properties under Euler discretization scheme.

The important problem in the systems' theory is the estimation of the unknown state of a system from outputs and inputs. This problem is known as "observing the state", hence the name "observer". Therefore the estimation of the unknown state is the task of the observer. In the construction of observers the outputs and inputs applied to the systems are used. In the last few decades state estimators (or observers) have been widely used in control and signal processing, see for instance [22, 23]. The problem of the observer synthesis for fractional discrete-time systems with Grünwald-Letnikov-type operator and the step

*e-mail: m.wyrwas@pb.edu.pl

Manuscript submitted 2016-11-08, revised 2017-01-31, initially accepted for publication 2017-02-23, published in December 2017. $h=1$ has been studied in $[24,25]$. The aim of the present paper is to study the construction of the full-order observers for linear fractional muti-order discrete-time systems with the RiemannLiouville- and Grünwald-Letnikov-type difference operators with the step $h>0$. We restrict the design of the observers for the systems whose fractional orders are from the interval $(0,1]$, because the systems with fractional orders higher than one can be always transform to systems with orders less than or equal to one, see for instance [26].

The paper is organized in the following way. Section 2 gathers preliminary notations, facts and definitions needed in the sequel. In Section 3 the initial value problems for fractional multi-order systems are presented. The main results of the paper, namely the construction of the fractional observer, that estimates the unknown state vector, is presented in Section 5. Since the fractional order system corresponding to the error vector should be asymptotically stable in order to guarantee the estimation of the unknown state of the system by the observer, the condition for asymptotic stability of fractional order systems is given in Section 4. Additionally, two examples that illustrate our results are presented. Finally, the conclusions are drawn.

\section{Preliminaries}

Let $h>0, a \in \mathbb{R}$ and $(h \mathbb{N})_{a}:=\{a, a+h, a+2 h, \ldots\}$. For a function $x:(h \mathbb{N})_{a} \rightarrow \mathbb{R}$ the forward $h$-difference operator is defined as (see [27]) $\left(\Delta_{h} x\right)(t):=\frac{x(t+h)-x(t)}{h}$, where $t \in(h \mathbb{N})_{a}$ and $\left(\Delta_{h}^{0} x\right)(t):=x(t)$. Let $k \in \mathbb{N}_{0}$ and $\Delta_{h}^{k}:=\Delta_{h} \circ \cdots \circ \Delta_{h}$ is $k$-fold application of operator $\Delta_{h}$. Then $\left(\Delta_{h}^{k} x\right)(t)=h^{-k} \sum_{i=0}^{k}(-1)^{k-i}\left(\begin{array}{l}k \\ i\end{array}\right) x(t+i h)$. Let us introduce the family of sequences on $\mathbb{Z}$ parameterized by $\mu>0$ and given by the values: $c^{(\mu)}(q)=\left(\begin{array}{c}q-\mu-1 \\ q\end{array}\right)=(-1)^{q}\left(\begin{array}{c}\mu \\ q\end{array}\right)$ for $q \in \mathbb{N}_{0}$ and $c_{\mu}(q)=0$ for $q<0$.

Definition 1. For a function $x:(h \mathbb{N})_{a} \rightarrow \mathbb{R}$ the fractional $h$-sum of order $\alpha>0$ is given by $\left({ }_{a} \Delta_{h}^{-\alpha} x\right)(t):=h^{\alpha}\left(c^{(-\alpha)} * \bar{x}\right)(q)$, where $t=a+q h, \bar{x}(q):=x(a+q h), q \in \mathbb{N}_{0}$ and "*" denotes a convolution operator, i.e. $\left(c^{(-\alpha)} * \bar{x}\right)(q):=\sum_{s=0}^{q}\left(\begin{array}{c}q-s+\alpha-1 \\ q-s\end{array}\right) \bar{x}(s)$. Additionally, we define $\left({ }_{a} \Delta_{h}^{0} x\right)(t):=x(t)$. 
For $\alpha=0$ we will write shortly $\Delta_{h}^{-\alpha}$ instead of ${ }_{0} \Delta_{h}^{-\alpha}$. Note that the fractional $h$-sum of order $\alpha$ does not change the domain of the function and ${ }_{a} \Delta_{h}^{-\alpha} x:(h \mathbb{N})_{a} \rightarrow \mathbb{R}$. Let us recall that the Z-transform of a sequence $\{y(q)\}_{q \in \mathbb{N}}$ is a complex function given by $Y(z):=\mathscr{Z}[y](z)=\sum_{q=0}^{\infty} y(q) z^{-q}$, where $z \in \mathbb{C}$ is a complex number for which the series $\sum_{q=0}^{\infty} y(q) z^{-q}$ converges absolutely. Then the inverse Z-transform addresses the reverse problem, i.e., given a function $Y(z)$ and a region of convergence, find the signal $y(q)$ whose Z-transform is $Y(z)$ and has the specified region of convergence. The presented $Z$-transform involves, by definition, only the values $y(q)$ of the sequence $\{y(q)\}_{q \in \mathbb{N}_{0}}$. Note that since $c^{(\alpha)}(q)=(-1)^{q}\left(\begin{array}{c}a \\ q\end{array}\right)$, then for $|z|>1$ we have

$$
\begin{aligned}
\mathscr{Z}\left[c^{(\alpha)}\right](z) & =\sum_{q=0}^{\infty}(-1)^{q}\left(\begin{array}{l}
\alpha \\
q
\end{array}\right) z^{-q}= \\
& =\sum_{g=0}^{\infty}\left(\begin{array}{c}
q-\alpha-1 \\
q
\end{array}\right) z^{-q}=\left(1-z^{-1}\right)^{\alpha}
\end{aligned}
$$

and

$$
\mathscr{Z}^{-1}\left[\left(1-z^{-1}\right)^{\alpha}\right]=c^{(\alpha)}
$$

The operator ${ }_{a} \Delta_{h}^{-\alpha}$ is defined as the convolution of two sequences, namely sequences $c^{(-\alpha)}$ and $\bar{x}$. Hence by the fact that the Z-transform of the convolution of two sequences is the product of the Z-transforms of the separate sequences one gets the following proposition.

Proposition 1. For $t=a+q h \in(h \mathbb{Z})_{a}$ let us define $y(q):=$ $:=\left({ }_{a} \Delta_{h}^{-\alpha} x\right)(t)$ and $\bar{x}(q):=x(a+q h)$. Then

$$
\mathscr{Z}[y](z)=h^{\alpha}\left(1-z^{-1}\right)^{-\alpha} X(z),
$$

where $X(z):=\mathscr{Z}[\bar{x}](z)$.

For $h=1$ the equation (3) can be rewritten as $\mathscr{Z}\left[{ }_{a} \Delta_{1}^{-\alpha} x\right](z)=$ $=\left(1-z^{-1}\right)^{-\alpha} X(z)$, where $\left({ }_{a} \Delta_{1}^{-\alpha} x\right)(a+q)=: y(q)$ is treated as a sequence.

Let $s \in \mathbb{N} a / h$ and $\tilde{x}: \mathbb{N} a / h \rightarrow \mathbb{R}$ be defined by $\tilde{x}(s):=x(s h)$. Then $s=a / h+q, q \in \mathbb{N}_{0}$ and $\bar{x}(q)=\tilde{x}(a / h+q)=x(a+q h)$ and of course $\bar{x}: \mathbb{N}_{0} \rightarrow \mathbb{R}$. Note that by (3) we get

$$
\mathscr{Z}[y]=h^{\alpha} \mathscr{Z}[\tilde{y}],
$$

where $\tilde{y}(q):=\left(a / h \Delta_{1}^{-\alpha} \tilde{x}\right)(a / h+q)$. Then using (4) one can easily proof the following lemma, that is also proven in [28] without using Z-transform method. This lemma gives the transition between fractional summation operators for any $h>0$ and $h=1$.

Lemma 1 [27]. Let $x:(h \mathbb{N})_{a} \rightarrow \mathbb{R}$ and $\alpha>0$. Then $\left({ }_{a} \Delta_{h}^{-\alpha} x\right)(t)=$ $=h^{\alpha}\left(a / h \Delta_{1}^{-\alpha} \tilde{x}\right)(t / h)$, where $t \in(h \mathbb{N})_{a}$ and $\tilde{x}(s)=x(s h)$.

For simplicity of notation if $h=1$, then we write: $a / h \Delta^{-\alpha}:=$ $:=a / h \Delta_{1}^{-\alpha}$.

2.1. Fractional difference operators and relation between them. Let us recall the definition of the Riemann-Liouville- and Grünwald-Letnikov-type $h$-difference operators and the forms of images in the Z-transform of the considered difference operators.

The definition of the Riemann-Liouville-type fractional $h$-difference operator can be found, for example, in [29] (for $h=1$ ) or in [27, 28] (for any $h>0$ ).

Definition 2. Let $\alpha \in(0,1]$. The Riemann-Liouville-type fractional $h$-difference operator ${ }_{a} \Delta_{h}^{\alpha}$ of order $\alpha$ for a function $x:(h \mathbb{N})_{a} \rightarrow \mathbb{R}$ is defined by $\left({ }_{a} \Delta_{h}^{\alpha} x\right)(t):=\left(\Delta_{h}\left({ }_{a} \Delta_{h}^{-(1-\alpha)} x\right)\right)(t)$, where $t \in(h \mathbb{N})_{a}$.

For the case $h=1$ we write: ${ }_{a} \Delta^{\alpha}:={ }_{a} \Delta_{1}^{\alpha}$.

Using the properties of $\mathrm{Z}$-transform the following proposition can be proven.

Proposition 2 [30]. For $a \in \mathbb{R}, \alpha \in(0,1]$ let us define $y(q):=$ $:=\left({ }_{a} \Delta_{h}^{\alpha} x\right)(t)$, where $t \in(h \mathbb{N})_{a}$ and $t=a+q h, q \in \mathbb{N}_{0}$. Then

$$
\mathscr{Z}[y](z)=z h^{-\alpha}\left(1-z^{-1}\right)^{\alpha} X(z)-z h^{-\alpha} x(a),
$$

where $X(z):=\mathscr{Z}[\bar{x}](z)$ and $\bar{x}(q):=x(a+q h)$.

For $\alpha=1$ we have $\mathscr{Z}[y](z)=1 / h((z-1) X(z)-z \bar{x}(0))$, that also agrees with the transform of difference $\Delta_{h}$ of $\bar{x}$.

Using Lemma 1 one can proof the transition formula for the Riemann-Liouville-type fractional $h$-difference operators between the cases for any $h>0$ and $h=1$, see for instance [27]. It is worth to stress that in [27] this formula is proven directly without using the Z-transform, but one can easily show it by using the $\mathrm{Z}$-transform method.

Lemma 2 [27]. Let $x:(h \mathbb{N})_{a} \rightarrow \mathbb{R}$ and $\alpha>0$. Then, $\left({ }_{a} \Delta_{h}^{\alpha} x\right)(t)=$ $=h^{-\alpha}\left(a / h \Delta^{\alpha} \tilde{x}\right)(t / h)$, where $t \in(h \mathbb{N})_{a}$ and $\tilde{x}(s)=x(s h)$.

The next type of the operator, that is considered, is the Grünwald-Letnikov-type fractional $h$-difference operator, see for example $[3,4,14,31-36]$ for cases $h=1$ and also for general case $h>0$.

Definition 3. Let $\alpha \in \mathbb{R}$. The Grünwald-Letnikov-type $h$-difference operator ${ }_{a} \widetilde{\Delta}_{h}^{\alpha}$ of order $\alpha$ for a function $x:(h \mathbb{N})_{a} \rightarrow \mathbb{R}$ is defined by

$$
\left({ }_{a} \widetilde{\Delta}_{h}^{\alpha} x\right)(t):=h^{-\alpha} \sum_{q=0}^{\frac{t-a}{h}} c^{(\alpha)}(q) x(t-q h) .
$$

For simplicity of the notation, if $h=1$, then we write: ${ }_{a} \widetilde{\Delta}^{\alpha}:={ }_{a} \widetilde{\Delta}_{1}^{\alpha}$. In [30] the following proposition is proven.

Proposition 3 [30]. For $a \in \mathbb{R}, \alpha \in(0,1]$ let us define $y(q):=$ $:=\left({ }_{a} \widetilde{\Delta}_{h}^{\alpha} x\right)(t)$, where $t \in(h \mathbb{N})_{a}$ and $t=a+q h, q \in \mathbb{N}_{0}$. Then

$$
\mathscr{Z}[y](z)=h^{-\alpha}\left(1-z^{-1}\right)^{\alpha} X(z),
$$

where $X(z):=\mathscr{Z}[\bar{x}](z)$ and $\bar{x}(q):=x(a+q h)$.

Observe that by (7) if $\bar{x}(q):=x(a+q h), q \in \mathbb{N}_{0}$, then one gets $\left({ }_{a} \widetilde{\Delta}_{h}^{\alpha} x\right)(a+q h)=h^{-\alpha}\left({ }_{0} \widetilde{\Delta}^{\alpha} \bar{x}\right)(q)$. 
By comparison of the formulas (5) and (7) one can show the relation between the Riemann-Liouville- and GrünwaldLetnikov-type fractional $h$-difference operator. The following proposition describes this relation and it has been proven in [30].

Proposition 4 [30]. Let $x:(h \mathbb{N})_{a} \rightarrow \mathbb{R}$. Then $\left({ }_{0} \widetilde{\Delta}_{h}^{\alpha} x\right)(t+h)=$ $=\left({ }_{a} \Delta_{h}^{\alpha} x\right)(t)$, for $t \in(h \mathbb{N})_{a}$.

It is well known that the Z-transform method can be used in finding the solutions of difference equations and in the study of the stability of their equilibrium points.

\section{Linear fractional multi-order difference systems}

In this section we consider initial value problems of fractional order systems of multi-order difference equations with the presented above types of operators. In fact the problem of solvability of fractional-order systems defined by difference equations with the Grünwald-Letnikov-type $h$-difference operator is discussed only, because the Riemann-Liouville-type $h$-difference operator can be expressed by the Grünwald-Letnikov-type operator, see Proposition 4. Solutions to initial value problems for control systems with all types of multi-order operators were stated in [37]. Let us consider the following initial value problem given by

$$
\begin{aligned}
\left({ }_{a} \widetilde{\Delta}_{h}^{\alpha_{i}} x_{i}\right)(t+h)= & \sum_{j=1}^{n} a_{i j} x_{j}(t)+\sum_{k=1}^{m} b_{i k} u_{k}(t), \\
& i=1, \ldots, n \\
x(a)= & {\left[\begin{array}{c}
x_{1}(a) \\
\vdots \\
x_{n}(a)
\end{array}\right] \in \mathbb{R}^{n} . }
\end{aligned}
$$

where $a \in \mathbb{R}, \alpha_{i} \in(0,1], h>0, x_{i}:(h \mathbb{N})_{a} \rightarrow \mathbb{R}$ is the state function, $t \in(h \mathbb{N})_{a}, a_{i j}, b_{i k} \in \mathbb{R}, i, j=1, \ldots, n, k=1, \ldots, m$, $n, m \in \mathbb{N}_{1}$, and $u_{k}:(h \mathbb{N})_{a} \rightarrow \mathbb{R}, k=1, \ldots, m$ is the input function applied to the system.

Of course, by Proposition 4 system (8a) can be equivalently written as

$$
\left({ }_{a} \Delta_{h}^{\alpha_{i}} x_{i}\right)(t)=\sum_{j=1}^{n} a_{i j} x_{j}(t)+\sum_{k=1}^{m} b_{i k} u_{k}(t), i=1, \ldots, n
$$

where the Riemann-Liouville-type $h$-difference operator is used instead of the Grünwald-Letnikov-type $h$-difference operator. Now, we restrict our considerations to the systems with the Grünwald-Letnikov-type difference operator, but for systems with Riemann-Liouville-type operator one gets the same results.

Using Lemma 2 the system (8a) can be rewritten in the form:

$$
\begin{aligned}
& \left({ }_{0} \widetilde{\Delta}^{\alpha_{i}} \bar{x}_{i}\right)(q+1)=h^{\alpha_{i}} \sum_{j=1}^{n} a_{i j} \bar{x}_{j}(q)+h^{\alpha_{i}} \sum_{k=1}^{m} b_{i k} \bar{u}_{k}(q), \\
& i=1, \ldots, n
\end{aligned}
$$

where $q \in \mathbb{N}_{0}, \bar{x}_{i}: \mathbb{N}_{0} \rightarrow \mathbb{R}, \bar{x}_{i}(q):=x_{i}(a+q h), i=1, \ldots, n$ and $\bar{u}_{j}: \mathbb{N}_{0} \rightarrow \mathbb{R}, \bar{u}_{j}(q):=u(a+q h), j=1, \ldots, m$. Moreover, the initial condition $(8 \mathrm{~b})$ can be rewritten as $\bar{x}(0)=\left(\bar{x}_{1}(0), \ldots\right.$, $\left.\bar{x}_{n}(0)\right)^{T} \in \mathbb{R}^{n}$.

Let $\bar{x}(q)=\left(\bar{x}_{1}(q), \ldots, \bar{x}_{n}(q)\right)^{T} \in \mathbb{R}^{n}$ and $\bar{u}(q)=\left(\bar{u}_{1}(q), \ldots\right.$, $\left.\bar{u}_{m}(q)\right)^{T} \in \mathbb{R}^{m}$ for $q \in \mathbb{N}_{0}$. Define ${ }_{0} \widetilde{\Delta}^{(\alpha)} \bar{x}:=\left({ }_{0} \widetilde{\Delta}^{\alpha_{1}} \bar{x}_{1}, \ldots,{ }_{0} \widetilde{\Delta}^{\alpha_{n}} \bar{x}_{n}\right)^{T}$. Then system (9) has the following matrix form

$$
\left({ }_{0} \widetilde{\Delta}^{(\alpha)} \bar{x}\right)(q+1)=H A \bar{x}(q)+H B \bar{u}(q),
$$

where $q \in \mathbb{N}_{0}, H:=\operatorname{diag}\left\{h^{\alpha_{1}}, \ldots, h^{\alpha_{n}}\right\} \in \mathbb{R}^{n \times n}, A=\left(a_{i j}\right) \in \mathbb{R}^{n \times n}$ and $B=\left(b_{i k}\right) \in \mathbb{R}^{n \times m}$. By Definition 3 it is easy to see that system (10) has the unique solution given by the recurrence formula

$$
\begin{aligned}
\bar{x}(q+1) & =H A \bar{x}(q)+H B \bar{u}(q)- \\
& -\sum_{i=1}^{q+1} \mathscr{C}^{(\alpha)}(i) \bar{x}(q-i+1),
\end{aligned}
$$

for any $q \in \mathbb{N}_{0}$, where $\mathscr{C}^{(\alpha)}(i):=\operatorname{diag}\left\{c^{\left(\alpha_{1}\right)}(i), \ldots, c^{\left(\alpha_{n}\right)}(i)\right\}$, $i=1, \ldots, q+1$, and consequently we have

$$
\begin{aligned}
x(a+(q+1) h) & =H A x(a+q h)+H B u(a+q h) \\
& -\sum_{i=1}^{q+1} \mathscr{C}^{(\alpha)}(i) x(a+(q-i+1) h),
\end{aligned}
$$

where $x(a+k h)=\left(x_{1}(a+k h), \ldots, x_{n}(a+k h)\right)^{T}$ for $k=0, \ldots$, $q+1$ and $u(a+k h)=\left(u_{1}(a+k h), \ldots, u_{m}(a+k h)\right)^{T}$ for $k=0, \ldots, q$.

Now, we show how the solutions of system (8a) look like in term of fundamental matrices associated to the considered systems. Let us define $y(q):=\left({ }_{0} \widetilde{\Delta}^{(\alpha)} \bar{x}\right)(q+1)$, where $q \in \mathbb{N}_{0}$ and

$$
\Lambda_{(\alpha)}:=\operatorname{diag}\left\{\left(1-z^{-1}\right)^{-\alpha_{1}}, \ldots,\left(1-z^{-1}\right)^{-\alpha_{n}}\right\} .
$$

Taking into account Proposition 3 and using the properties of Z-transform one gets the following formula:

$$
\mathscr{Z}[y](z)=z H^{-1} \Lambda_{(-\alpha)} X(z)-z H^{-1} \bar{x}(0),
$$

where $X(z)=\left(\mathscr{Z}\left[\bar{x}_{1}\right](z), \ldots, \mathscr{Z}\left[\bar{x}_{n}\right](z)\right)^{T}$. Note that $H^{-1}=\operatorname{diag}\left\{h^{-\alpha_{1}}\right.$, $\left.\ldots, h^{-\alpha_{n}}\right\}$ and $\Lambda_{(-\alpha)}=\Lambda_{(\alpha)}^{-1}=\operatorname{diag}\left\{\left(1-z^{-1}\right)^{\alpha_{1}}, \ldots,\left(1-z^{-1}\right)^{\alpha_{n}}\right\}$.

The solutions of the fractional difference system (8a) with initial conditions (8b) can be expressed by the fundamental matrices associated to the considered systems.

Proposition 5. Let $\alpha_{i} \in(0,1], i=1, \ldots, n$ and $\Phi$ be the fundamental matrix for system (8a) such that $x(t)=\Phi\left(\frac{t-a}{h}\right) x(a)+$ $+\sum_{i=0}^{\frac{t-a}{h}-1} \Phi\left(\frac{t-a}{h}-i-1\right) H B u(a+i h)$ is the solution to the initial value problem (8). Then

$$
\mathscr{Z}[\Phi](z)=\left(I-z^{-1} \Lambda_{(\alpha)} H A\right)^{-1} \Lambda_{(\alpha)}=\left(\Lambda_{(-\alpha)}-z^{-1} H A\right)^{-1},
$$

where $\Lambda_{(\alpha)}=\operatorname{diag}\left\{\left(1-z^{-1}\right)^{-\alpha_{1}}, \ldots,\left(1-z^{-1}\right)^{-\alpha_{n}}\right\}, H=\operatorname{diag}\left\{h_{1}^{\alpha_{1}}\right.$, $\left.\ldots, h_{n}^{\alpha_{n}}\right\}$. 
Proof. Note that for $\bar{x}(q)=x(a+q h)$ and $\bar{u}(q)=u(a+q h)$ system (8a) can be written in the equivalent matrix form (10). The idea of the proof is to take the Z-transform of each equation of system (10) and use formula (13). Then we get the system of algebraic equations:

$$
z H^{-1} \Lambda_{(-\alpha)} X(z)-z H^{-1} \bar{x}(0)=A X(z)+B U(z),
$$

where $U(z)=\left(\mathscr{Z}\left[\bar{u}_{1}\right](z), \ldots, \mathscr{Z}\left[\bar{u}_{m}\right](z)\right)^{T}$. Hence one gets

$$
\left(z I-\Lambda_{(\alpha)} H A\right) X(z)=z \Lambda_{(\alpha)} \bar{x}(0)+\Lambda_{(\alpha)} H B U(z),
$$

for $\bar{x}(0)=x(a)=\left[x_{1}\left(a_{1}\right) \ldots x_{n}\left(a_{n}\right)\right]^{T}$. Then

$$
\begin{aligned}
X(z)= & \left(I-z^{-1} \Lambda_{(\alpha)} H A\right)^{-1} \Lambda_{(\alpha)} x(a)+ \\
& +z^{-1}\left(I-z^{-1} \Lambda_{(\alpha)} H A\right)^{-1} \Lambda_{(\alpha)} H B U(z)= \\
= & \mathscr{Z}[\Phi](z) x(a)+\mathscr{Z}[\Phi * H B u](z) .
\end{aligned}
$$

Hence

$$
\bar{x}(q)=\Phi(q) \bar{x}(0)+\sum_{i=0}^{q-1} \Phi(q-1-i) H B \bar{u}(i)
$$

Consequently, since an initial condition is arbitrary, we get the thesis.

Remark 1. Note that $\Lambda_{(\alpha)}=\Lambda_{(-\alpha)}^{-1}$ and

$$
\begin{aligned}
\left(I-z^{-1} \Lambda_{(\alpha)} H A\right)^{-1} \Lambda_{(\alpha)} & =\left(I-z^{-1} \Lambda_{(\alpha)} H A\right)^{-1} \Lambda_{(-\alpha)}^{-1} \\
& =\left(\Lambda_{(-\alpha)}-z^{-1} H A\right)^{-1}
\end{aligned}
$$

Hence for $h=1$ the result coincides with Proposition 3 given in $[26]$.

\section{Stability of fractional multi-order systems}

In this section we state the asymptotic stability conditions of fractional multi-order difference systems of the form (8a).

In the proof of Proposition 5 we give the $z$-domain solution to the state equations (8a), which are equivalently rewritten in the matrix form as (10) and we get

$$
\begin{aligned}
X(z)= & \left(I-z^{-1} \Lambda_{(\alpha)} H A\right)^{-1} \Lambda_{(\alpha)} x(a)+ \\
& +\left(z \Lambda_{(-\alpha)}-H A\right)^{-1} H B U(z)= \\
= & z^{-1}\left(z \Lambda_{(-\alpha)}-H A\right)^{-1} x(a) \\
& +\left(z \Lambda_{(-\alpha)}-H A\right)^{-1} B U(z),
\end{aligned}
$$

where $\Lambda_{(\alpha)}=\operatorname{diag}\left\{\left(1-z^{-1}\right)^{-\alpha_{1}}, \ldots,\left(1-z^{-1}\right)^{-\alpha_{n}}\right\}, H=\operatorname{diag}\left\{h^{\alpha_{1}}\right.$, $\left.\ldots, h^{\alpha_{n}}\right\}$.
Proposition 6. Let $R$ be the set of all roots of the equation

$$
\operatorname{det}\left(I-z^{-1} \Lambda_{(\alpha)} H A\right)=0
$$

Then the following items are satisfied.

a) If all elements from $R$ are strictly inside the unit circle, then system (8a) is asymptotically stable.

b) If there is $z \in R$ such that $|z|>1$, then system (8a) is not stable.

Proof. The proof is similar to those presented in [30]. Here the proof is based on the formula of Z-transform of function $\Phi(\cdot)$ from Proposition 5, see formula (14).

Remark 2. In fact, the $z$-domain solution of (8a), under the condition $x_{0}=0$, is as follows

$$
X(z)=\left(z \Lambda_{(-\alpha)}-H A\right)^{-1} H B U(z)
$$

Observe that the set of the roots of the equation (15) equals the set of the roots of the following equation

$$
\operatorname{det}\left(z \Lambda_{(-\alpha)}-H A\right)=0
$$

Therefore, for $\alpha_{1}, \ldots, \alpha_{n} \in(0,1]$ if all roots of the equation (16) are strictly inside the unit circle, then (8a) is asymptotically stable.

\section{Observers}

In this section we present the construction of the full-order observer and formulate the conditions that guarantee the existence of observers.

Let us consider the following linear control systems (8a) with the output given by:

$$
y(t)=C x(t)+D u(t)
$$

where $t \in(h \mathbb{N})_{a}, y:(h \mathbb{N})_{a} \rightarrow \mathbb{R}^{p}, C \in \mathbb{R}^{p \times n}$ and $D \in \mathbb{R}^{p \times m}$.

Definition 4. The following system

$\left({ }_{0} \widetilde{\Delta}^{(\beta)} \widehat{x}\right)(q+1)=F \widehat{x}_{j}(q)+G u(a+q h)+K y(a+q h)$,

is called a (fractional) observer of order $(\beta)=\left(\beta_{1}, \ldots, \beta_{n}\right)$ for system (8a) with output (17) if

$$
\lim _{q \rightarrow \infty} e(q)=\lim _{q \rightarrow \infty}(x(a+q h)-\hat{x}(q))=0,
$$

for any $x(a), \hat{x}(0)$ and $u:(h \mathbb{N})_{a} \rightarrow \mathbb{R}$, where $\widehat{x}: \mathbb{N}_{0} \rightarrow \mathbb{R}^{n}$, $\widehat{x}:=\left[\widehat{x}_{1}, \ldots, \widehat{x}_{n}\right]^{T}, e:=\left[e_{1}, \ldots, e_{n}\right]^{T}: \mathbb{N}_{0} \rightarrow \mathbb{R}^{n}$ is an error vector such that $e_{i}:=\bar{x}_{i},-\widehat{x}_{i}, F \in \mathbb{R}^{n \times n}, G \in \mathbb{R}^{n \times m}$ and $K \in \mathbb{R}^{n \times p}$. 
Then $\hat{x}_{i}(q)$ estimates the unknown value $x_{i}(a+q h)$ of the state $x$ and $\hat{x}$ is called an estimation of $x$.

Observe that if $\beta_{i}=1, i=1, \ldots, n$, in Definition 4 , then an observer is a classical delta-difference system. Taking $\beta_{i} \in(0,1]$ one gets observers being fractional systems with commensurate and incommensurate orders.

Now, let us formulate the condition that guarantees the existence of the observer. Assume that $(\beta)=(\alpha)$, i.e. $\beta_{i}=\alpha_{i}$ for $i=1, \ldots, n$.

Theorem 7. If there exists matrix $K$ such that all roots of the equation

$$
\operatorname{det}\left(I-z^{-1} \Lambda_{(\alpha)}(H A-K C)\right)=0,
$$

are strictly inside the unit circle, then the observer for the system (8a) is as follows:

$$
\begin{aligned}
\left({ }_{0} \widetilde{\Delta}^{(\alpha)} \widehat{x}\right)(q+1) & =(H A-K C) \hat{x}(q)+ \\
& +H B \bar{u}(q)+K \bar{y}(q)
\end{aligned}
$$

where $\bar{y}(q)=y(a+q h)$ and $\bar{u}(q)=u(a+q h)$.

Proof. Note that (8a) can be rewritten in the equivalent matrix form (10). By (10) and (21) for the error function $e: \mathbb{N}_{0} \rightarrow \mathbb{R}^{n}$, one gets

$$
\begin{aligned}
\left({ }_{0} \widetilde{\Delta}^{(\alpha)} e\right)(q+1)= & \left({ }_{0} \widetilde{\Delta}^{(\alpha)} \bar{x}\right)(q+1)-\left({ }_{0} \widetilde{\Delta}^{(\alpha)} \hat{x}\right)(q+1)= \\
= & H A \bar{x}(q)+H B \bar{u}(q)-H A \widehat{x}(q)-H B \bar{u}(q)- \\
& -K(\bar{y}(q)-C \widehat{x}(q)) \\
= & H A(\bar{x}(q)-\widehat{x}(q))-K(\bar{y}(q)-C \widehat{x}(q)) .
\end{aligned}
$$

Since $\bar{y}(q)=y(a+q h)=C x(a+q h)+\operatorname{Dux}(a+q h)=C \bar{x}(q)+$ $+D \bar{u}(q)$ and $e(q)=\bar{x}(q)-\widehat{x}(q)$, we have

$$
\left({ }_{0} \widetilde{\Delta}^{(\alpha)} e\right)(q+1)=(H A-K C) e(q)-K D \bar{u}(q)
$$

By assumption all roots of the equation (20) are strictly inside the unit circle, so using Proposition 6 we get the asymptotic stability of (22). Then $\lim _{q \rightarrow \infty} e(q)=\lim _{q \rightarrow \infty}(\bar{x}(q)-\widehat{x}(q))=0$ and the system (21) is the observer for system (8a) with output (17).

Remark 3. Note that if only all roots of the equation (20) are strictly inside the unit circle, then the observer (21) estimates all coordinates of the unknown state $x$. Hence both $x$ and the estimate $\hat{x}$ are from $\mathbb{R}^{n}$. Therefore in order to design the fractional observer for a linear fractional multi-order difference system one has to find a matrix $K$ that satisfies the assumption of Theorem 7.

Now, let us consider a numerical example that illustrates the behaviour of the constructed observer.

Example 1. Let us consider the fractional system defined by (8a) and (17) with $\alpha_{1}=0.2, \alpha_{2}=0.6, h=0.5$ and matrices:

$A=\left[\begin{array}{cc}0.6 & -0.3 \\ 0 & -0.4\end{array}\right], B=\left[\begin{array}{l}b_{1} \\ b_{2}\end{array}\right], C=\left[\begin{array}{ll}1 & 1\end{array}\right]$ and $D=d$ where $b_{1}, b_{2}$, $d \in \mathbb{R}$. Then $\Lambda_{\alpha}=\left[\begin{array}{cc}\left(\frac{z}{z-1}\right)^{0.2} & 0 \\ 0 & \left(\frac{z}{z-1}\right)^{0.6}\end{array}\right]$ and $H=\left[\begin{array}{cc}0.5^{0.2} & 0 \\ 0 & 0.5^{0.6}\end{array}\right]$. Our task is to find the matrix $K=\left[\begin{array}{l}a \\ b\end{array}\right] \in \mathbb{R}^{2 \times 1}$ such that all roots of the equation (20) are strictly inside the unit circle.

Taking $a=0.5$ and $b=-0.1$ we get that all roots of equation (20) (i.e. the complex numbers $0.9799925913 e-1$ $\pm 0.1070545235 i$ and $|0.9799925913 e-1 \pm 0.1070545235 i|=$ $=0.1451362318$ computed by Maple) are strictly inside the unit circle, so the following system

$$
\begin{aligned}
\left({ }_{0} \widetilde{\Delta}^{0.2} \widehat{x}_{1}\right)(q+1)= & 0.5^{0.2} \cdot 0.6 \widehat{x}_{1}(q)-0.5^{0.2} \cdot 0.3 \widehat{x}_{2}(q)+ \\
& +0.5^{0.2} \cdot b_{1} \bar{u}(q)+0.5(\bar{y}(q)- \\
& \left.-\widehat{x}_{1}(q)-\widehat{x}_{2}(q)\right) \\
\left({ }_{0} \widetilde{\Delta}^{0.6} \widehat{x}_{1}\right)(q+1)= & -0.5^{0.6} \cdot 0.4 \widehat{x}_{2}(q)+0.5^{0.6} \cdot b_{2} \bar{u}(q)- \\
& -0.1\left(\bar{y}(q)-\widehat{x}_{1}(q)-\widehat{x}_{2}(q)\right),
\end{aligned}
$$

where $\bar{u}(q)=u(0.5 q)$ and $\bar{y}(q)=y(0.5 q), q \in \mathbb{N}_{0}$, is an observer of the considered systems, i.e. the following equivalent systems:

$$
\begin{aligned}
\left({ }_{0} \Delta_{0.5}^{0.2} \widehat{x}_{1}\right)(0.5 q)= & 0.6 x_{1}(0.5 q)-0.3 x_{2}(0.5 q)+ \\
& +b_{1} u(0.5 q) \\
\left({ }_{0} \Delta_{0.5}^{0.6} \widehat{x}_{2}\right)(0.5 q)= & 0.4 x_{2}(0.5 q)+b_{2} u(0.5 q) \\
y(0.5 q)= & x_{1}(0.5 q)+x_{2}(0.5 q)+d u(0.5 q)
\end{aligned}
$$

and

$$
\begin{aligned}
\left({ }_{0} \widetilde{\Delta}_{0.5}^{0.2} \widehat{x}_{1}\right)(0.5(q+1))= & 0.6 x_{1}(0.5 q)-0.3 x_{2}(0.5 q)+ \\
& +b_{1} u(0.5 q) \\
\left({ }_{0} \widetilde{\Delta}_{0.5}^{0.6} \widehat{x}_{2}\right)(0.5(q+1))= & 0.4 x_{2}(0.5 q)+b_{2} u(0.5 q) \\
y(0.5 q)= & x_{1}(0.5 q)+x_{2}(0.5 q)+d u(0.5 q)
\end{aligned}
$$

with the Riemann-Liouville-type and with the Grünwald-Letnikov-type operators, respectively. 
(a) the graphs of $x_{1}$ and $\hat{x}_{1}=y_{1}$ for $n=50$ steps

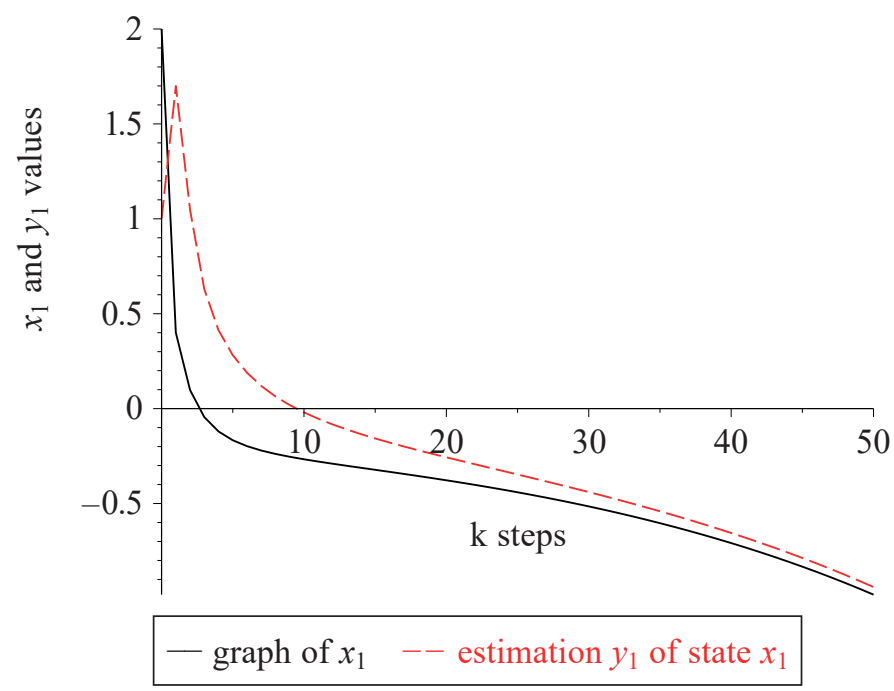

(b) the graphs of $x_{2}$ and $\widehat{x}_{2}=y_{2}$ for $n=50$ steps

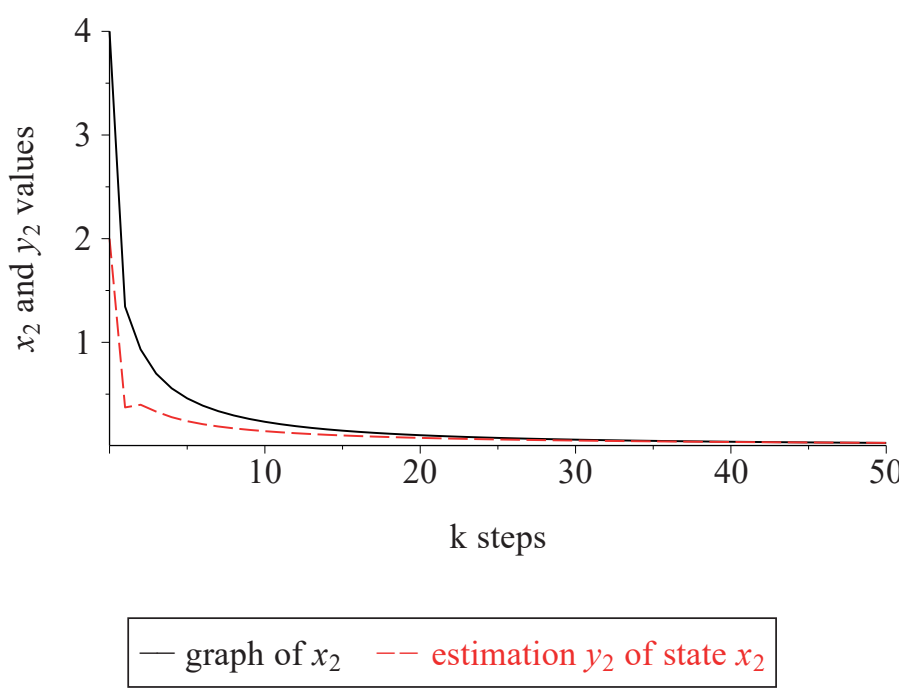

Fig. 1. The solution of the initial value problem for the systems (24) (or equivalently (25)) and (23) in Example 1

The observer (23) estimates the state of the original systems (24) and (25). Figure 1 shows the behaviour of the observer (23) with initial conditions $\widehat{x}_{1}(0)=1$ and $\widehat{x}_{2}(0)=2$ and the behaviour of the original systems's solution with initial conditions $x_{1}(0)=2$ and $x_{2}(0)=4$ for $u \equiv 0$. For the better visualization the points are connected.

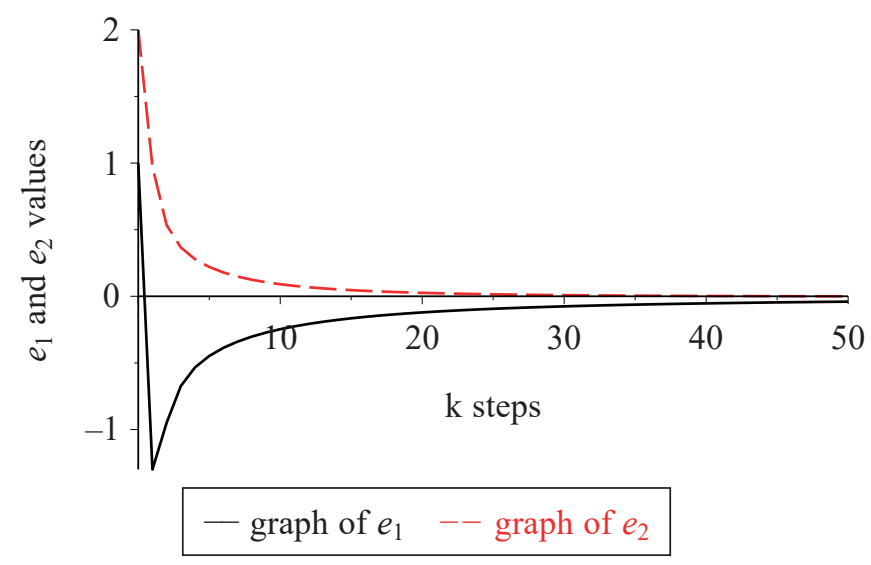

Fig. 2. The trajectory corresponding to the initial value problem for the system (22) with the initial error's conditions $e_{1}(0)=1, e_{2}(0)=2$

Observe that Fig. 1 shows the graphs of the states and their estimations while Fig. 2 illustrates the behaviour of the error function, where $e_{i}:=x_{i}-\hat{x}_{i}, i=1,2$.

Now, we present the construction of the observer for discrete-time model obtained in [38] for ultracapacitor discrete time identification. Our goal is to find the estimation of the unknown state of the given discrete-time model basing on the knowledge of output and input.
Example 2. Let us consider the fractional system with the Grünwald-Letnikov-type operator defined by (8a) and (17) with $\alpha_{1}=\alpha_{2}=0.5, h=1$ and matrices:

$$
\begin{aligned}
& A=\left[\begin{array}{cc}
0 & 1 \\
-0.006333 & -0.037401
\end{array}\right], B=\left[\begin{array}{l}
0 \\
1
\end{array}\right], \\
& C=\left[\begin{array}{ll}
0.025055 & 0.004997
\end{array}\right] \text { and } D=[0.223395] .
\end{aligned}
$$$$
\text { Then } \Lambda_{\alpha}=\left[\begin{array}{cc}
\left(\frac{z}{z-1}\right)^{0.5} & 0 \\
0 & \left(\frac{z}{z-1}\right)^{0.5}
\end{array}\right] \text { and } H=I \text {. }
$$

Observe that taking for instance $K=\left[\begin{array}{l}30 \\ 10\end{array}\right] \in \mathbb{R}^{2 \times 1}$ all roots of equation (20) are strictly inside the unit circle, so the following system:

$$
\begin{aligned}
\left({ }_{0} \widetilde{\Delta}^{0.5} \widehat{x}_{1}\right)(q+1) & =\widehat{x}_{2}(q) \\
\left({ }_{0} \widetilde{\Delta}^{0.5} \widehat{x}_{2}\right)(q+1) & =-0.006333 \widehat{x}_{1}(q)- \\
& -0.037401 \widehat{x}_{2}(q)+u(q),
\end{aligned}
$$

where $q \in \mathbb{N}_{0}$, is an observer of the following system:

$$
\begin{aligned}
\left({ }_{0} \widetilde{\Delta}^{0.5} \widehat{x}_{1}\right)(q+1) & =\widehat{x}_{2}(q) \\
\left({ }_{0} \widetilde{\Delta}^{0.5} \widehat{x}_{2}\right)(q+1) & =-0.006333 x_{1}(q)- \\
& -0.037401 x_{2}(q)+u(q) \\
y(q) & =0.025055 x_{1}(q)+0.004997 x_{2}(q)+ \\
& +0.223395 u(q) .
\end{aligned}
$$

The observer (26) estimates the state of the system (27). Figure 3 shows the behaviour of the observer (26) with initial 
(a) the graphs of $x_{1}$ and $\widehat{x}_{1}=y_{1}$ for $n=50$ steps

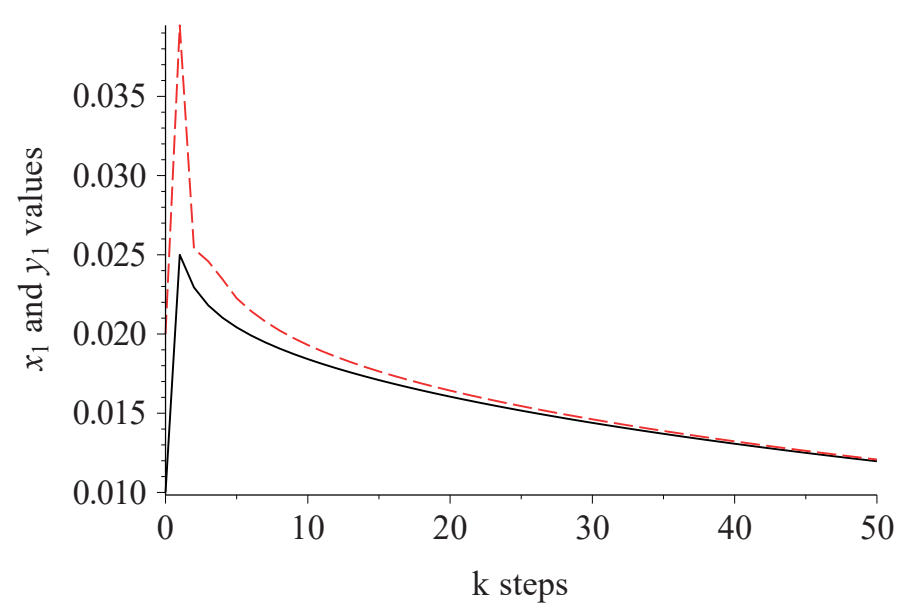

- graph of $x_{1} \quad--$ estimation $y_{1}$ of state $x_{1}$ (b) the graphs of $x_{2}$ and $\widehat{x}_{2}=y_{2}$ for $n=50$ steps

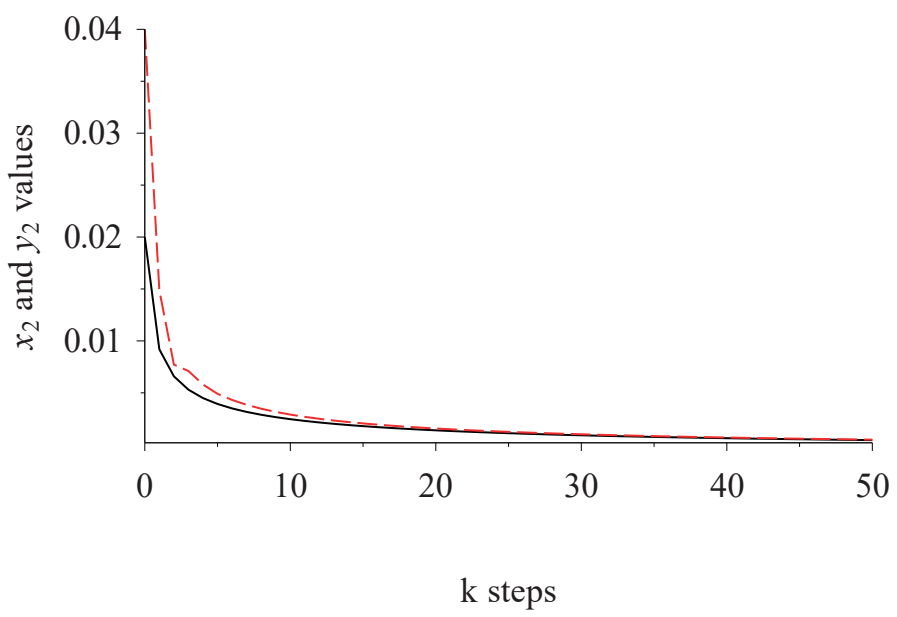

- graph of $x_{2}--$ estimation $y_{2}$ of state $x_{2}$

Fig. 3. The solution of the initial value problem for the systems (27) and (26) in Example 2

conditions $\widehat{x}_{1}(0)=0.02$ and $\widehat{x}_{2}(0)=0.04$ and the behaviour of the original systems's solution with initial conditions $x_{1}(0)=0.01$ and $x_{2}(0)=0.02$ for $u \equiv 0$. Again for the better visualization the points are connected. The behaviour of the error function is illustrated by Fig. 1 .

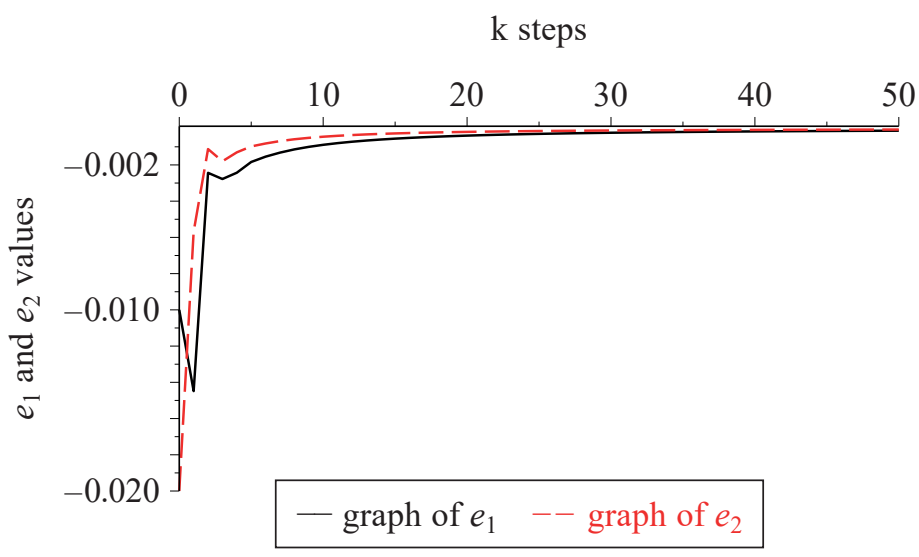

Fig. 4. The trajectory corresponding to the initial value problem for the system (22) with the initial error's conditions $e_{1}(0)=-0.01$,

$$
e_{2}(0)=-0.02
$$

\section{Conclusions}

In the paper the construction of fractional observers for multiorder fractional difference systems with orders $\alpha_{i} \in(0,1]$, $i=1, \ldots, n$ is presented. The condition that guarantees the existence of the fractional observer is given. The construction of the presented fractional observer requires to find the gain matrix $K$ that satisfies the condition that guarantees the asymptotic stability of the fractional multi-order system related with the error vector. Our future goal will be devoted to formulate and prove the ultimate analytical stability criteria for the fractional multi-order systems. Additionally, we will study the existence of observers for the difference systems with the Caputo-type operators.

Acknowledgements. The work was financed by the Bialystok University of Technology grant No. S/WI/1/2016.

\section{REFERENCES}

[1] L. Chen, W. Pan, R. Wu, and Y. He, "New result on finitetime stability of fractional-order nonlinear delayed systems", Journal of Computational and Nonlinear Dynamics 10 (6), 5 pages (2015).

[2] J. Gabano and T. Poinot, "Fractional modelling and identification of thermal systems", Signal Processing 91, 531-541 (2011).

[3] T. Kaczorek and P. Ostalczyk, "Responses comparison of the two discrete-time linear fractional state-space models", Fractional Calculus and Applied Analysis 19 (4), 789-805, (2016).

[4] T. Kaczorek, "A new approach to the realization problem for fractional discrete-time linear systems", Bull. Pol. Ac.: Tech. 64 (1), 9-14, 2016.

[5] T. Kaczorek, Selected Problems of Fractional Systems Theory, Springer, 2011.

[6] D. Mozyrska, E. Girejko, and M. Wyrwas, "Fractional nonlinear systems with sequential operators", Central European Journal of Physics 11 (10), 1295-1303 (2013).

[7] D. Mozyrska and E. Pawluszewicz, "Local controllability of nonlinear discrete-time fractional order systems", Bull. Pol. Ac.: Tech. 61 (1), 251-256 (2013).

[8] D. Mozyrska, E. Pawłuszewicz, and M.Wyrwas, "Local observability and controllability of nonlinear discrete-time fractional order systems based on their linearisation", International Journal of Systems Science 48 (4), 788-794, 2017. 
[9] D. Mozyrska and M. Wyrwas, "Fractional discrete-time of Hegselmann-Krause's type consensus model with numerical simulations", Neurocomputing 216, 381-392 (2016).

[10] P. Ostalczyk, Discrete Fractional Calculus: Applications in Control and Image Processing, Series in Computer Vision - Volume 4, World Scientific Publishing Co, Singapore, 2016.

[11] D. Sierociuk, T. Skovranek, M. Macias, I. Podlubny, I. Petras, A. Dzielinski, and P. Ziubinski, "Diffusion process modeling by using fractional-order models", Applied Mathematics and Computation 257, 2-11 (2015)

[12] R. Stanisławski, M. Rydel, and K.J. Latawiec, "Modeling of discrete-time fractional-order state space systems using the balanced truncation method", Journal of the Franklin Institute 354(7), 3008-3020, (2017).

[13] A.A. Kilbas, H. M. Srivastava, and J.J. Trujillo, Theory and Applications of Fractional Differential Equations, North-Holland Mathematics Studies, 204. Elsevier Science B. V., Amsterdam, 2006.

[14] I. Podlubny, Fractional Differential Equations, Academic Press, San Diego-Boston-New York-London-Tokyo-Toronto, 1999.

[15] K.S. Miller and B. Ross, "Fractional difference calculus", In Proceedings of the International Symposium on Univalent Functions, Fractional Calculus and their Applications, pages 139-152, Kōriyama, Japan, 1988. Nihon University.

[16] T. Abdeljawad and F. M. Atıc1, "On the definitions of nabla fractional operators", Abstract and Applied Analysis 2012, 13 pages (2012).

[17] F.M. Atıc1 and P.W. Eloe, "Discrete fractional calculus with the nabla operator", Electronic Journal of Qualitative Theory of Differential Equations Spec. Ed. I 2009 (3), 1-12 (2009).

[18] F. Chen, X. Luo, and Y. Zhou, "Existence results for nonlinear fractional difference equation", Advances in Difference Equations 2011, 12 pages (2011).

[19] D. Mozyrska and E. Girejko, "Overview of the fractional $h$-difference operators", In Frank-Olme Speck Alexandre Almeida, Luis Castro, editor, Operator Theory: Advances and Applications, volume 229, pages 253-267. Birkhäuser, 2013.

[20] M.D. Ortigueira, F.J.V. Coito, and J.J. Trujillo, "Discretetime differential systems", Signal Processing 107, 198-217 (2015).

[21] S.R. Anderson and V. Kadirkamanathan, "Modelling and identification of nonlinear deterministic systems in deltadomain", Automatica 43, 1859-1868 (2007).

[22] M. Boutayeb, M. Darouach, and H. Rafaralahy, "Generalized state-space observers for chaotic synchronization and secure communication", IEEE Transactions on Circuits and Systems, I: Fundamental Theory and Applications 49 (3), 345-349 (2002).

[23] M. Darouach, M. Zasadzinski, and S. Xu, "Full-order observers for linear systems with unknown inputs", IEEE Transactions on Automatic Control 39 (3), 606-609 (1994).
[24] A. Dzieliński and D. Sierociuk, "Observer for discrete fractional order state-space systems", 2nd IFAC Workshop on Fractional Diffrentation and its Applications, IFAC FDA '06, Portugal, 2006, 524-529.

[25] D. Sierociuk, "Estimation and control of discrete-time dybnamical fractional systems described in state space", Ph.D. thesis, Warsaw University of Technology, Warsaw 2007.

[26] M. Wyrwas and D. Mozyrska, Theoretical Developments and Applications of Non-Integer Order Systems, In Stefan Domek and Paweł Dworak, editors, Lecture Notes in Electrical Engineering, volume 357, chapter: "Stability of linear discrete- time systems with fractional positive orders", 157-166, Springer, 2015.

[27] R.A.C. Ferreira and D.F.M. Torres, "Fractional $h$-difference equations arising from the calculus of variations", Applicable Analysis and Discrete Mathematics 5 (1), 110-121 (2011).

[28] N.R.O. Bastos, R.A.C. Ferreira, and D.F.M. Torres, "Discrete-time fractional variational problems", Signal Processing 91 (3), 513-524 (2011).

[29] F.M. Atıc1 and P.W.Eloe, "A transform method in discrete fractional calculus", International Journal of Difference Equations 2, 165-176 (2007).

[30] D. Mozyrska and M. Wyrwas, "The Z-transform method and delta type fractional difference operators", Discrete Dynamics in Nature and Society 2015, 12 pages (2015).

[31] M. Busłowicz, "Robust stability of positive discrete-time linear systems of fractional order", Bull. Pol. Ac.: Tech. 58 (4), 567-572 (2010).

[32] M. Busłowicz and T. Kaczorek, "Simple conditions for practical stability of positive fractional discrete-time linear systems", International Journal of Applied Mathematics and Computer Science 19 (2), 236-269 (2009).

[33] M. Busłowicz and A. Ruszewski, "Necessary and sufficient conditions for stability of fractional discrete-time linear statespace systems", Bull. Pol. Ac.: Tech. 61 (4), 779-786 (2013).

[34] D. Sierociuk and A. Dzieliński, "Stability of discrete fractional order state-space systems", Journal of Vibration and Control 14 (9-10), 1543-1556 (2008).

[35] R. Stanisławski and K.J. Latawiec, "Stability analysis for discrete-time fractional-order LTI state-space systems. Part I: New necessary and sufficient conditions for the asymptotic stability", Bull. Pol. Ac.: Tech. 61 (2), 353-361, (2013).

[36] R. Stanisławski and K.J. Latawiec, "Stability analysis for discrete-time fractional-order LTI state-space systems. Part II: New stability criterion for FD-based systems", Bull. Pol. Ac.: Tech. 61 (2), 363-370, (2013).

[37] D. Mozyrska, "Multiparameter fractional difference linear control systems", Discrete Dynamics in Nature and Society, 2014, 8 pages (2014)

[38] A. Dzieliński, D. Sierociuk, and G. Sarwas, "Some applications of fractional order calculus", Bull. Pol. Ac.: Tech. 58 (4), 583-592 (2010). 